Twist grain boundaries in cubic surfactant phases

Maxim Belushkin, and Gerhard Gompper

Citation: The Journal of Chemical Physics 130, 134712 (2009); doi: 10.1063/1.3096987

View online: https://doi.org/10.1063/1.3096987

View Table of Contents: http://aip.scitation.org/toc/jcp/130/13

Published by the American Institute of Physics

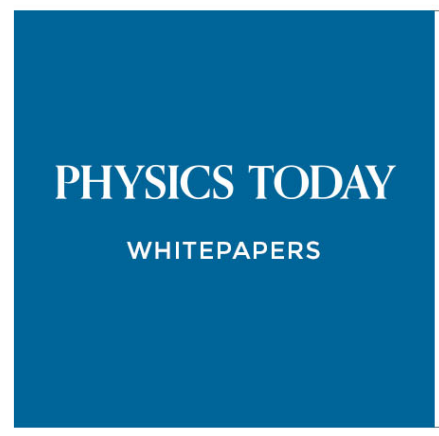
systems can do 


\title{
Twist grain boundaries in cubic surfactant phases
}

\author{
Maxim Belushkin ${ }^{\text {a) }}$ and Gerhard Gompper ${ }^{\text {b) }}$ \\ Institut für Festkörperforschung, Forschungszentrum Jülich, D-52425 Jülich, Germany
}

(Received 19 December 2008; accepted 18 February 2009; published online 7 April 2009)

\begin{abstract}
Twist grain boundaries in bicontinuous cubic surfactant phases are studied by employing a Ginzburg-Landau model of ternary amphiphilic systems. Calculations are performed on a discrete real-space lattice with periodic boundary conditions for the lamellar $L_{\alpha}$, gyroid $G$, diamond $D$, and the Schwarz $P$ phases for various twist angles. An isosurface analysis of the scalar order parameter reveals the structure of the surfactant monolayer at the interfaces between the oil-rich and water-rich regions. The curvature distributions show that the grain boundaries are minimal surfaces. The interfacial free energy per unit area is determined as a function of the twist angle for the $G, D, P$, and lamellar phases using two complementary approaches: the Ginzburg-Landau free-energy functional and a geometrical approach based on the curvature energy of a monolayer. For the $L_{\alpha}, G$, and $D$ phases the interfacial free energy per unit area is very small, has the same order of magnitude, and exhibits a nonmonotonic dependence on the twist angle. The $P$ phase is found to be unstable with respect to the nucleation of grain boundaries. (C) 2009 American Institute of Physics.
\end{abstract}

[DOI: 10.1063/1.3096987]

\section{INTRODUCTION}

Amphiphilic molecules added to an immiscible oil-water system self-assemble into a large variety of structures. ${ }^{1-9}$ Phases with cubic symmetry often feature a triply-periodic minimal surface (TPMS) configuration of the surfactant monolayer. ${ }^{4-6,10-12}$ Examples of such phases include the gyroid $G$, diamond $D$, Schwarz $P$, Schoen $I-W P, F-R D$, Neovius $C(P)$, and others, which are encountered in physical systems. Properties of cubic surfactant phases have been a subject of extensive theoretical $^{11-16}$ and experimental ${ }^{4-6}$ interests, with applications in biological systems, ${ }^{6,17}$ as templates for mesoporous systems ${ }^{18}$ and for the crystallization of membrane proteins. ${ }^{19}$ Cubic surfactant phases are liquid crystalline structures $^{20}$ with the fundamental building block-the unit cell-repeating itself in all directions. The lattice constant in amphiphilic systems is large, of the order of $10 \mathrm{~nm}$.

In amphiphilic systems many kinds of interfaces occur: between two ordered phases, between ordered and disordered phases, and between two grains of the same ordered phase which differ by their spacial orientation. Recently, interfaces between coexisting lamellar, hexagonal, and disordered phases, ${ }^{21-23}$ interfaces between lamellar and gyroid phases, ${ }^{24,25}$ dislocations in cubic phases, ${ }^{26}$ and grain boundaries in the lamellar phase $e^{22,27,28}$ have been studied. Twist grain boundaries are interfaces between two grains of the same phase which differ only by a rotation around an axis perpendicular to the grain boundary. Twist grain boundaries in the lamellar phase have been investigated within self-consistent field theory for diblock copolymers ${ }^{28}$ and

\footnotetext{
${ }^{a)}$ Electronic mail: m.belushkin@fz-juelich.de.

${ }^{b)}$ Electronic mail: g.gompper@fz-juelich.de.
}

observed experimentally ${ }^{29}$ to have a structure similar to Scherk's minimal surface. Tilt grain boundaries in the lamellar phase, where the normals of the lamellae of the two grains define a plane perpendicular to the boundary, have been shown within a Ginzburg-Landau theory to be chevron-shaped at small tilt angles and omega-shaped at large tilt angles, ${ }^{22,27}$ in agreement with experimental results. ${ }^{30}$ The nucleation of defects in the gyroid cubic mesophase has recently been observed in Lattice-Boltzmann simulations. $^{31}$

We investigate here twist grain boundaries in cubic surfactant phases. The calculations are based on a GinzburgLandau theory with a single scalar order parameter $\phi(\vec{r})$, which describes the local oil-water concentration difference. $^{32}$ The geometrical properties of the grain boundaries are evaluated on the isosurface $\phi(\vec{r}) \equiv 0$, which defines the position of the surfactant monolayer. The interfacial free energy per unit area of a grain boundary depends on the angles with respect to the crystalline axes. It is determined using the Ginzburg-Landau theory and a complementary geometrical approach based on the CanhamHelfrich curvature Hamiltonian. For simplicity, we use the notation "interfacial tension" in the remainder of this paper.

The paper is organized as follows. First, we introduce the elements of the Ginzburg-Landau theory used to describe the cubic surfactant phases. In Sec. III we describe the computational methods. Section IV deals with the methods of analysis of the order parameter configurations obtained from the Ginzburg-Landau theory and presents the methods used to extract the interfacial tension. Our results for the twist grain boundaries in the lamellar $L_{\alpha}$ and the cubic $G, D$, and $P$ phases are presented in Sec. V. 


\section{THEORY}

\section{A. Ginzburg-Landau theory}

A Ginzburg-Landau theory of ternary amphiphilic systems, which successfully describes their phase behavior and mesoscopic structure, was proposed in Ref. 32. The model contains a single scalar order parameter $\phi(\vec{r})$, which describes the local oil-water concentration difference. The amphiphilic degrees of freedom are considered to be integrated out in this approach. ${ }^{33}$ The model is based on the free-energy functional

$$
\mathcal{F}[\phi]=\int d \vec{r}\left[(\Delta \phi)^{2}+g(\phi)(\nabla \phi)^{2}+f(\phi)\right] .
$$

In order to obtain three-phase coexistence, the function $f(\phi)$ is taken to be $\mathrm{b}^{34}$

$$
f(\phi)=(\phi+1)^{2}(\phi-1)^{2}\left(\phi^{2}+f_{0}\right),
$$

where the three minima at $\phi=-1,0,+1$ correspond to excess water, microemulsion, and excess oil phases, respectively. The parameter $f_{0}$ acts as a chemical potential for amphiphiles. The surfactant monolayer is given by the isosurface $\phi(\vec{r}) \equiv 0$. The function $g(\phi)$ has the form ${ }^{34}$

$$
g(\phi)=g_{0}+g_{2} \phi^{2} .
$$

Here the parameters $g_{0}<0$ and $g_{2}>0$ are related to the amphiphilic strength and the solubility of the amphiphile. ${ }^{7}$

Alternatively, the elastic properties of the amphiphilic monolayer can be described by the Canham-Helfrich Hamiltonian $^{35,36}$

$$
\mathcal{H}=\int d A\left[\sigma+2 \kappa\left(H-c_{0}\right)^{2}+\bar{\kappa} K\right],
$$

where $\sigma$ is the surface tension, $\kappa$ is the bending rigidity, $\bar{\kappa}$ is the saddle-splay modulus, $c_{0}$ is the spontaneous curvature, and $H$ and $K$ are the mean and Gaussian curvatures, respectively. The integration in Eq. (4) extends over the surfactant monolayer. Due to the oil-water symmetry in balanced microemulsions, the spontaneous curvature $c_{0}$ vanishes.

Approaches based on the Canham-Helfrich Hamiltonian (4) and on the Ginzburg-Landau free-energy functional (1) have been shown to be related, and the elastic constants $\kappa$ and $\bar{\kappa}$ of the curvature energy have been calculated in terms of the parameters of the Ginzburg-Landau theory. ${ }^{7,37,38}$

\section{B. Cubic surfactant phases}

The Ginzburg-Landau theory has been successfully used to describe a large number of triply periodic minimal surface structures of the surfactant monolayer using real-space ${ }^{39}$ and Fourier-space ${ }^{13}$ representations. The TPMS configurations arise as local minima of the free-energy functional (1). However, the cubic phases are only metastable with respect to the lamellar phase for the parameter values investigated in Ref. 13. Since we use the same parameter values as Ref. 13, the cubic phases are also metastable in our calculations.

In the present analysis, we generate the gyroid $G$, diamond $D$, and Schwarz $P$ structures by initializing $\phi(\vec{r})$ by the leading order term of the Fourier expansion and minimizing

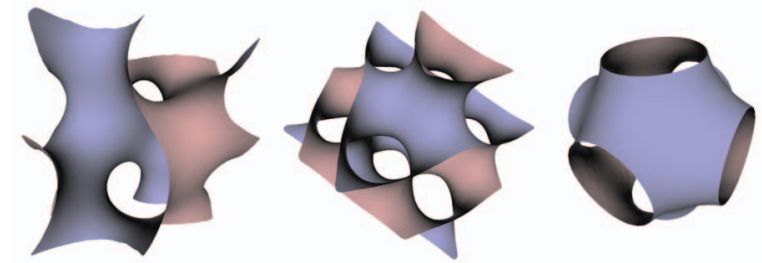

FIG. 1. (Color) Structure of the gyroid $G$ (left), diamond $D$ (center), and Schwarz $P$ (right) minimal surface configurations obtained by minimizing the free-energy functional (1).

the free energy (1) with respect to the order parameter and the unit cell size $a$. Following Ref. 13, we fix the parameters of the Ginzburg-Landau theory as $f_{0}=0.0, g_{0}=-3.0$, and $g_{2}=7.01$.

The resulting unit cells are shown on Fig. 1. The unit cell sizes of the $G, D$, and $P$ configurations are $a_{G}=10.9$, $a_{D}=13.2$, and $a_{P}=7.6$, respectively, in good agreement with the values obtained from the Fourier analysis in Ref. 13.

\section{CALCULATION METHOD}

We follow Ref. 40 for the discretization of the free energy (1) on a $N \times N \times N_{z}$ spatial lattice. The number of lattice sites per unit cell is fixed to $N_{u}=40$ for the lamellar, gyroid $G$, and diamond $D$ phases, and to $N_{u}=20$ for the $P$ phase.

First, we generate lattices containing bulk phases twisted by angles $\alpha / 2$ in the $x y$ plane. This is achieved by using the leading order Fourier expressions ${ }^{41}$ to initialize the lattices in real space. Since we keep the resolution per unit cell fixed, the lattice size $N$ depends on the twist angle $\alpha / 2$ to satisfy periodic boundary conditions. Simulations can only be performed for angles at which the rotated structure is periodic in the $x y$ plane of size $N \times N$, with $N$ sufficiently small to keep computational time reasonable (see Table I below).

The free energy is then minimized with respect to the order parameter to achieve equilibrium configurations. The minimization of the free-energy functional (1) with respect to the discretized field $\phi_{i j k}$, where $i j k$ represent indices of the discrete lattice sites, is based on the method of gradient descent. During the calculation, random lattice sites $i j k$ are selected and the gradient $d F / d \phi_{i j k}$ is computed locally. An adjustment $\phi_{i j k} \rightarrow \phi_{i j k}-\beta_{i j k} d F / d \phi_{i j k}$ is attempted. The matrix $\beta_{i j k}$ is adaptively adjusted: if the adjustment minimizes the local contribution of the lattice site $i j k$ to the total free

TABLE I. List of angles and phases in which corresponding twist grain boundaries have been calculated. The computation box dimensions are the same for the $L_{\alpha}, G$, and $D$ phases and smaller for the $P$ phase for which we have chosen a smaller resolution per unit cell.

\begin{tabular}{lcc}
\hline \hline $\begin{array}{l}\text { Twist angle } \\
(\mathrm{deg})\end{array}$ & $\begin{array}{c}\text { Calculated } \\
\text { in phases }\end{array}$ & $\begin{array}{c}\text { Box dimension } \\
N\left(L_{\alpha}, G, D / P\right)\end{array}$ \\
\hline 22.6 & $L_{\alpha}, G, P$ & $204 / 103$ \\
27.8 & $L_{\alpha}, G, D, P$ & $165 / 83$ \\
36.8 & $P$ & $\cdots / 64$ \\
53.0 & $L_{\alpha}, G, D, P$ & $89 / 45$ \\
67.2 & $L_{\alpha}, G, D$ & $144 / \cdots$ \\
73.8 & $L_{\alpha}, G, P$ & $200 / 101$ \\
90.0 & $L_{\alpha}, G, D, P$ & $113 / 29$ \\
\hline
\end{tabular}




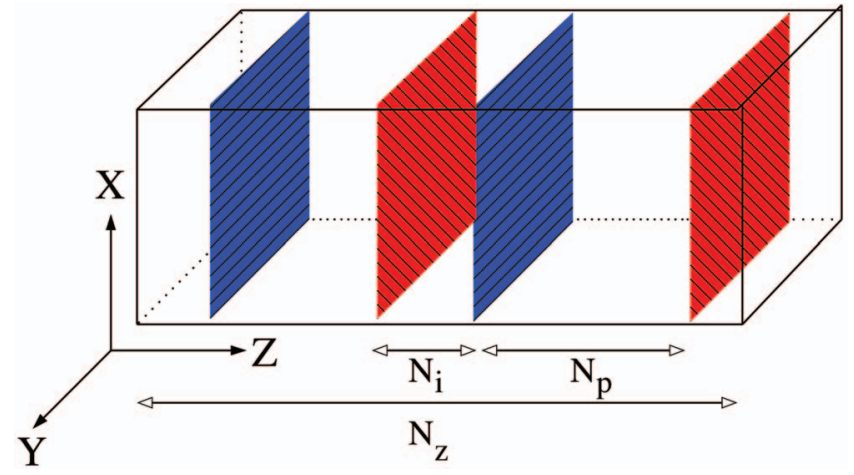

FIG. 2. (Color) A schematic view of the computation box. $N_{i}$ denotes the thickness of the grain boundary at the end of the calculation and $N_{p}$ corresponds to the thickness of the bulk-phase regions not affected by the grain boundaries as determined by the similarity integral (5).

energy (1), $\beta_{i j k}$ is increased; if the change in $\phi_{i j k}$ does not decrease the free energy, $\beta_{i j k}$ is decreased. In total, more than 10000 adjustments per lattice site are made to reach an equilibrium configuration. The gradient descent method approaches the solution asymptotically. Our stopping criteria are based on the average magnitude of the values in the line search matrix $\left\langle\beta_{i j k} d F / d \phi_{i j k}\right\rangle$ being of the order of $10^{-8}$.

We obtain a very good agreement with the Fourier analysis in Ref. 13 for the free-energy densities and unit cell sizes for the lamellar $G$ and $D$ phases and good agreement for the $P$ phase where discretization effects play a more pronounced role due to fewer lattice sites $N_{u}$ per unit cell.

The bulk-phase lattices are then combined to produce the grain-boundary system. Given a fixed size $N_{z}$ in the $z$ direction, half of the box is filled with an equilibrated bulk phase rotated by an angle $-\alpha / 2$ and the second half with an equilibrated bulk phase rotated by an angle $+\alpha / 2$. Periodic boundary conditions are imposed in the $x, y$, and $z$ directions. The $z$ direction is perpendicular to the grain boundaries. Due to periodic boundary conditions, two grain boundaries develop-one in the middle of the box between the bulkphase regions and one at both ends of the box in the $z$ direction. A schematic view of the system is given in Fig. 2. The choice of the box size $N_{z}$ in the $z$ direction is discussed in Sec. V A.

\section{ANALYSIS OF COMPUTATION RESULTS}

\section{A. Location and geometry of the grain boundaries}

In order to quantitatively determine the locations of the grain boundaries, we relate the final result for the order parameter configuration to the original bulk phases by a similarity integral

$$
\mathcal{S}(z)=\frac{\int d x d y\left(\phi_{f}-\phi_{i}\right)^{2}}{\int d x d y \phi_{i}^{2}},
$$

where $\phi_{i}$ corresponds to the initial bulk phases and $\phi_{f}$ corresponds to the final result of free-energy minimization. The integral vanishes if and only if $\phi_{i}=\phi_{f}$, which corresponds to preserved bulk-phase regions. Nonzero values of $\mathcal{S}(z)$ correspond to grain boundaries.
The surfactant monolayer corresponding to the isosurface $\phi \equiv 0$ is extracted as a triangulated surface using the GNU Triangulated Surface Library. ${ }^{42}$ For each phase and twist angle, the distributions of the mean square $H^{2}$ and Gaussian $K$ curvatures on the isosurface are extracted. The curvature distributions are then compared between the bulkphase and grain-boundary regions and further analyzed using the Canham-Helfrich curvature Hamiltonian (4).

\section{B. Determination of the interfacial tension}

We determine the interfacial tension $\Sigma$ using two complementary approaches.

\section{Interfacial tension from the Ginzburg-Landau theory}

The first approach is a direct calculation using the Ginzburg-Landau free-energy functional (1),

$$
2 \Sigma=\frac{F-F_{\text {bulk }}}{A},
$$

where $A$ is the area of the grain boundary and $F$ and $F_{\text {bulk }}$ are the free energies of the full system and the bulk-phase regions of equal size, respectively. The factor 2 in Eq. (6) arises from the presence of two grain boundaries due to periodic boundary conditions.

\section{Interfacial tension from geometrical considerations}

Geometrical approaches are based on the CanhamHelfrich Hamiltonian (4) for the isosurface corresponding to the surfactant monolayer. We will show in Sec. V below that the surfactant monolayer in the grain boundaries is very well described by a minimal surface. Therefore, the meancurvature squared term $H^{2} d S$ in Eq. (4) vanishes. We adopt an approach similar to Ref. 20 to extend the CanhamHelfrich Hamiltonian to include a higher-order term. Since the mean curvature nearly vanishes, the next nonzero contribution arises from the squared Gaussian-curvature term, so that

$$
\mathcal{H}=\int d A\left[\sigma+\bar{\kappa} K+\overline{\bar{\kappa}} K^{2}\right] .
$$

First, we consider the isosurface $\phi \equiv 0$ as a fluid membrane. In this case, the surface tension term $\sigma d A$ in Eq. (7) can be neglected. ${ }^{20}$ For minimal surfaces the Gaussian curvature $K \leq 0$ on the whole surface. The requirement that minimal surfaces of nonplanar configuration be stable leads to $\bar{\kappa}>0$ such that the second term in Eq. (7) is negative. In order for the Hamiltonian (7) to be stable with respect to the creation of infinitely narrow necks $(K \rightarrow-\infty)$ the last term has to be positive, thus $\overline{\bar{\kappa}}>0$. For a fixed value of $\bar{\kappa}$, the value of $\overline{\bar{\kappa}}$ is determined by the length scale of the unit cell such that the cubic phases are local minima of the Hamiltonian (7). The Hamiltonian in this approach is then given by

$$
\mathcal{H}_{1}=\int d A\left[\bar{\kappa} K+\overline{\bar{\kappa}} K^{2}\right],
$$

where $\bar{\kappa}>0$ and $\overline{\bar{\kappa}}>0$. 


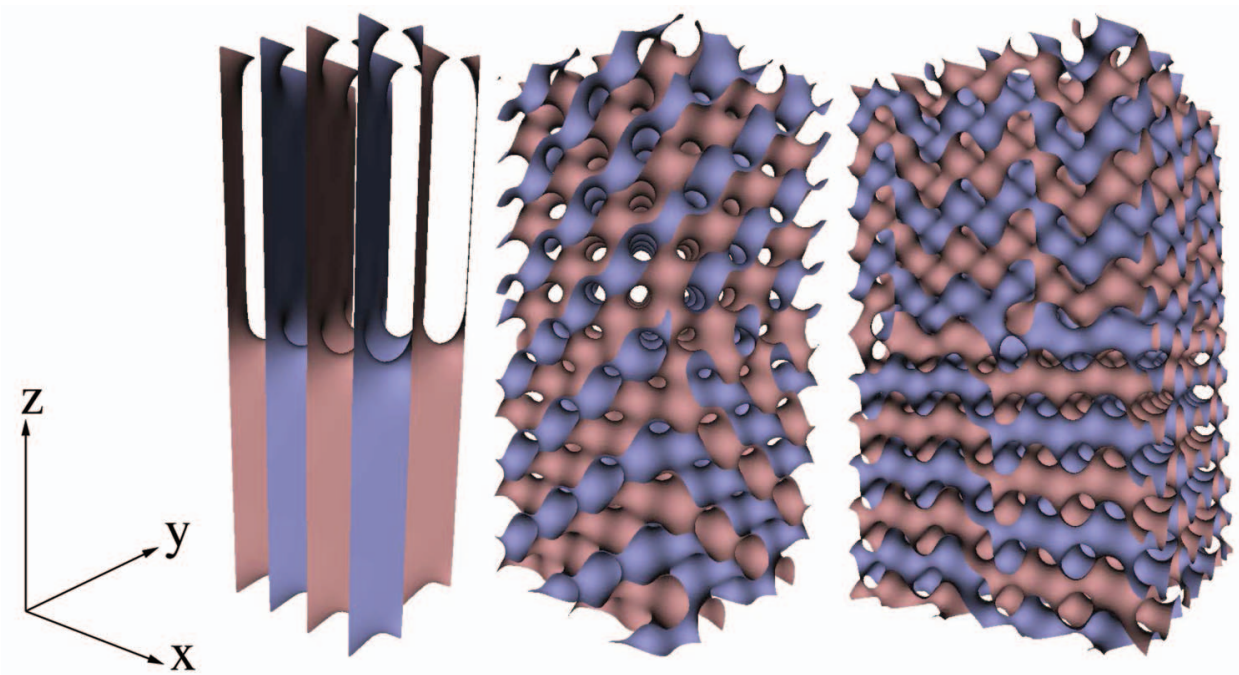

FIG. 3. (Color) Configuration of the surfactant monolayer for the lamellar phase at a twist angle of $53^{\circ}$ (left), gyroid phase at a twist angle of $90^{\circ}$ (center), and diamond phase at a twist angle of $67.2^{\circ}$ (right). The grain boundaries are located in the middle and at the top/bottom of each box in the $z$ direction.
On the other hand, it has been shown that the GinzburgLandau model and the model based on the Canham-Helfrich Hamiltonian (4) are related, and the elastic bending moduli have been derived from the Ginzburg-Landau theory. ${ }^{37,38}$ For $g_{0}<0, g_{2}>0$, and $f_{0}$ close to zero, the calculation yields $\sigma<0, \kappa>0$, and $\bar{\kappa}<0$. Here $\sigma$ corresponds to the chemical potential of the amphiphile. The value of $\sigma$ sets the normalization of the Hamiltonian (7). We choose $\sigma=-1.0$ and find the value of the saddle-splay modulus $\kappa_{0}$, which minimizes the Hamiltonian (7) for the cubic phases with $\overline{\bar{\kappa}}=0$. However, the curvature energy is very insensitive to the geometry of the monolayer in this case because it only depends on the total area and the Euler characteristic, both of which are topological invariants. Sensitivity to the monolayer geometry arises from the $\overline{\bar{\kappa}} K^{2} d S$ term. In order to obtain a nonzero value for $\overline{\bar{\kappa}}$, we set $\bar{\kappa}=\bar{\kappa}_{0}-\delta \bar{\kappa}$ and determine $\overline{\bar{\kappa}}$ by the requirement that the cubic phases are local minima of the Hamiltonian (7) with the new value $\bar{\kappa}$. In our calculations we choose the ratio $\delta \bar{\kappa} / \bar{\kappa}_{0}=0.3$, so that $|\bar{\kappa}|<\left|\bar{\kappa}_{0}\right|$. The Hamiltonian in this approach is thus given by

$$
\mathcal{H}_{2}=\int d A\left[\sigma+\bar{\kappa} K+\overline{\bar{\kappa}} K^{2}\right],
$$

where $\sigma<0, \bar{\kappa}<0$, and $\overline{\bar{\kappa}}>0$.

\section{RESULTS}

We have performed calculations of twist grain boundaries in the $L_{\alpha}, G, D$, and $P$ phases for a variety of twist angles listed in Table I. Figure 3 shows the configuration of the surfactant monolayer in the full computation boxes for the lamellar, gyroid, and diamond phases at different twist angles.

\section{A. Grain boundary geometry}

The similarity integral (5) is used to quantitatively determine the locations of the grain boundaries. The results for the $G$ phase at a twist angle of $67.2^{\circ}$ and the $D$ phase at a twist angle of $22.6^{\circ}$ are shown in Fig. 4. For all angles in the $G$ and $D$ phases we observe the grain-boundary thickness to be about $1 \mathrm{u}$ cell of the bulk phase. This property of the grain boundaries to preserve the length scales of the bulk phases was observed in initial calculations for the $G$ phase in which $N_{z}$ was varied between $4.5 N_{u}$ and $6 N_{u}$, where $N_{u}=40$ is the number of lattice sites per unit cell in the $z$ direction. Therefore, in all our calculations we use a thickness $N_{i}$ of one unit cell for the grain boundaries and a thickness $N_{p}$ of two unit cells for the bulk phases. Thus, $N_{z}=2 N_{p}+2 N_{i}=6 N_{u}$ (see Fig. 2).

In the lamellar, $G$, and $D$ phases, the positions of the grain boundaries are well determined. The behavior of the $P$ phase is different. The similarity integrals for the $P$ phase for twist angles of $22.6^{\circ}$ and $73.8^{\circ}$ are shown in Fig. 5. Significant modifications in the original bulk regions occur. In what follows for the $P$ phase, we define the grain boundaries as layers $1 \mathrm{u}$ cell thick in the $z$ direction around the maximum peaks of the similarity integral and the regions of two unit cell thickness in between as the bulk regions. The initial $P$ phase, rotated by an angle $\alpha / 2$ used to initialize the system, is called the original bulk. A comparison of the geometry of the original bulk $P$ phase rotated by $\alpha / 2=26.5^{\circ}$ and the final bulk region is shown in Fig. 6. Significant deviations are evident, including a rotation and distortion of the central unit cell.

The full isosurface $\phi \equiv 0$ is separated in the $z$ direction into the bulk and grain-boundary regions. The grain-

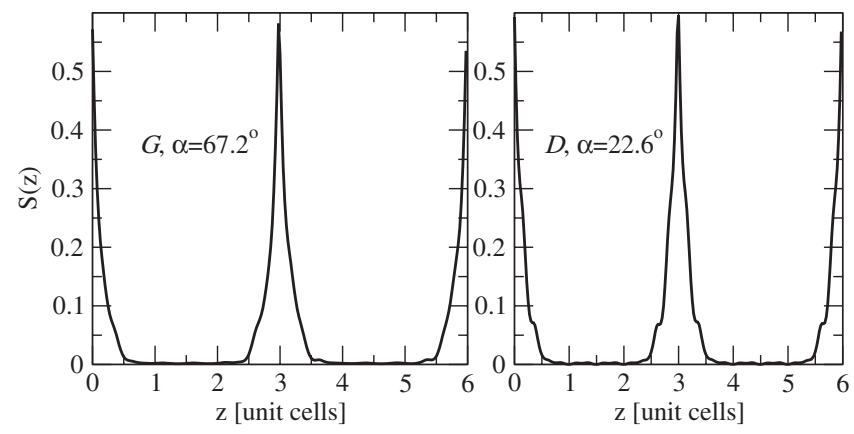

FIG. 4. Similarity integral $\mathcal{S}(z)$ for the $G$ phase at a twist angle of $67.2^{\circ}$ (left) and the $D$ phase at a twist angle of $22.6^{\circ}$ (right). Regions of nonzero values of $\mathcal{S}(z)$ indicate the positions of the grain boundaries. Both grain boundaries in the middle and at the top/bottom in the $z$ direction are well resolved and have a thickness of about $1 \mathrm{u}$ cell. 


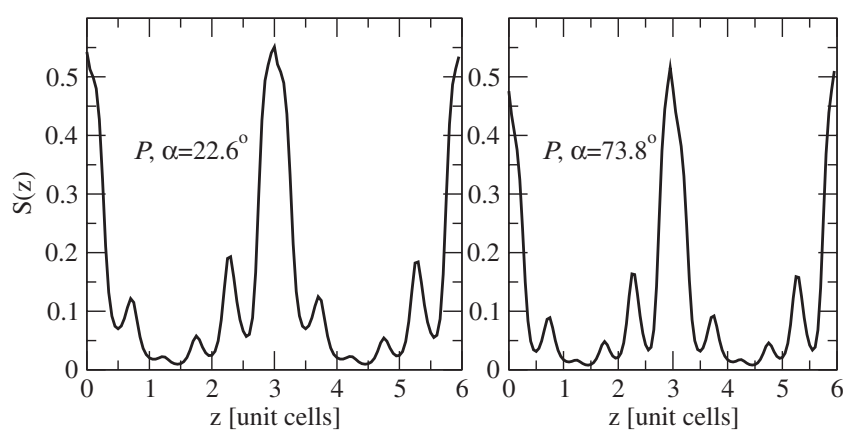

FIG. 5. Similarity integral for the $P$ phase at twist angles of $22.6^{\circ}$ (left) and $73.8^{\circ}$ (right). The bulk is significantly modified by the presence of the grain boundaries.

boundary regions are taken to be $1 \mathrm{u}$ cell thick layers of the box centered around the maxima of the similarity integral (5). The geometry of the grain boundaries for the lamellar, gyroid, diamond, and Schwarz $P$ phases for twist angles of $53^{\circ}$ and $90^{\circ}$ is shown in Fig. 7.

The mean-curvature and Gaussian-curvature distributions for the bulk and grain-boundary regions are calculated using the routines in the GTS Library. ${ }^{42}$ Curvature histograms are given in terms of area fractions corresponding to small curvature intervals. The normalization of all histograms is such that the sum of all bin values is unity.

The squared-mean-curvature distributions for the $L_{\alpha}, G$, $D$, and $P$ phases for several twist angles $\alpha$ are shown in Fig. 8. Discretization errors lead to a nonvanishing area fraction of nonzero mean curvature in all cases except the trivial case of planar geometries in the lamellar phase. The discretization effects can be estimated by comparing squared-meancurvature distributions of the discretized minimal-surface unit cells shown in Fig. 1 and spherical caps of different radii generated on a lattice of the same size. The resulting squared-mean-curvature distributions, shifted by the spontaneous curvature $c_{0}$, are shown in Fig. 9. The spontaneous curvature $c_{0}$ vanishes for the lamellar and gyroid geometries, and $c_{0}=1 / R$ for the spherical caps. A zero-width distribution is achieved only for the trivial case of planar geometry. For spherical caps the distribution becomes wider as the curvature increases. The gyroid unit cell with a characteristic radius $R_{G}=1 / \sqrt{\langle K\rangle} \approx a / 5$ has the broadest distribution since it has the most complex geometry. All distributions have their maxima at $\left(H-c_{0}\right)^{2}=0$.
It is therefore only meaningful to compare the curvature distributions in the grain-boundary regions to the corresponding curvature distributions in the bulk. Figure 8 shows the comparison of the squared-mean-curvature distributions of the bulk and grain-boundary regions for several twist angles for all the phases studied. Only in the bulk of the $L_{\alpha}$ phase are discretization errors negligible due to the trivial geometry. The squared-mean-curvature distributions in the grain-boundary regions in the $L_{\alpha}$ phase are consistent with $H^{2}=0$. An analysis of the grain-boundary geometry (Fig. 7, top) shows that the configurations of the monolayer are very similar to the Scherk's minimal surface. This result is in agreement with previous investigations of twist grain boundaries in the lamellar phase in diblock copolymer systems. ${ }^{28,29}$

In the $G$ and $D$ phases the squared-mean-curvature distributions in the grain-boundary regions compared to the corresponding bulk distributions are also consistent with $H^{2}=0$. The distributions in the grain-boundary regions are slightly broader than in the bulk. In our approach more complex geometries lead to larger variance in the curvature distributions. The monolayer in the grain-boundary regions has a more complex structure than in the bulk, which is composed of repetitions of the same unit cell.

The similarity integrals for the $P$ phase show that the bulk is greatly affected by the presence of grain boundaries. As we will show, the $P$ phase is unstable with respect to the nucleation of grain boundaries at the chosen point in the phase diagram. We nonetheless study the curvature distributions in the bulk and grain-boundary regions (Fig. 8, bottom). The squared-mean-curvature distributions (Fig. 8) in both the bulk and the grain-boundary regions are consistent with $H^{2}=0$.

The Gaussian-curvature distributions for the $G$ and $D$ phases for several twist angles are shown in Fig. 10. In the bulk regions all important features of the exact curvature distributions obtained from the Weierstrass representation ${ }^{13}$ are reproduced. However, discretization errors lead to a wider peak in the curvature distributions. Nevertheless, the configurations of the monolayer in the bulk regions are good discrete representations of the corresponding minimal surfaces.

In the lamellar, $G$, and $D$ phases, the geometry of the monolayer in the grain-boundary regions is clearly different from the bulk. In the $G$ and $D$ phases the maxima of the $K$

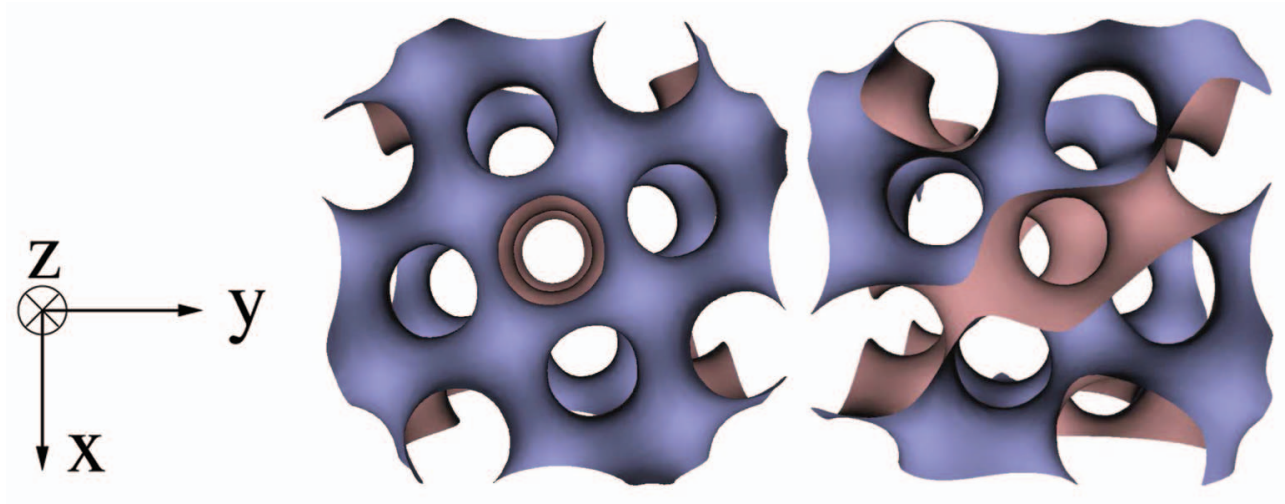

FIG. 6. (Color) Original phase (left) and section of the computation box between grain boundaries (right) for the $P$ phase at a twist angle of $\alpha=53.0^{\circ}$. 


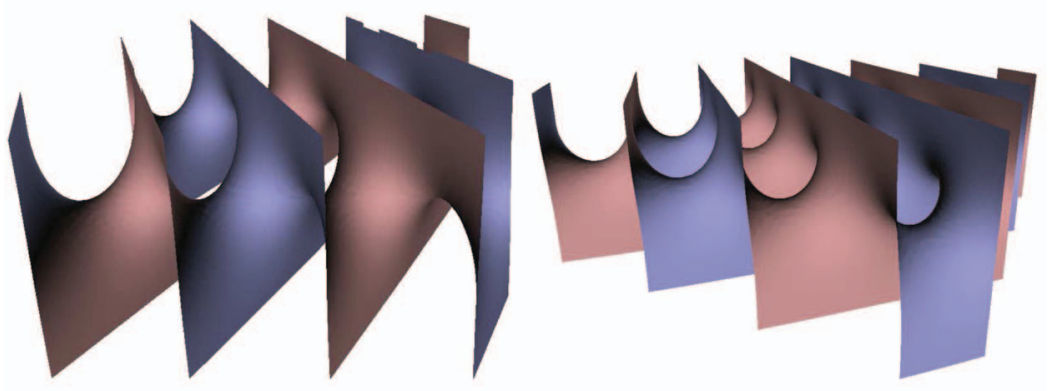

$L_{\alpha}, 53.0^{\circ}$
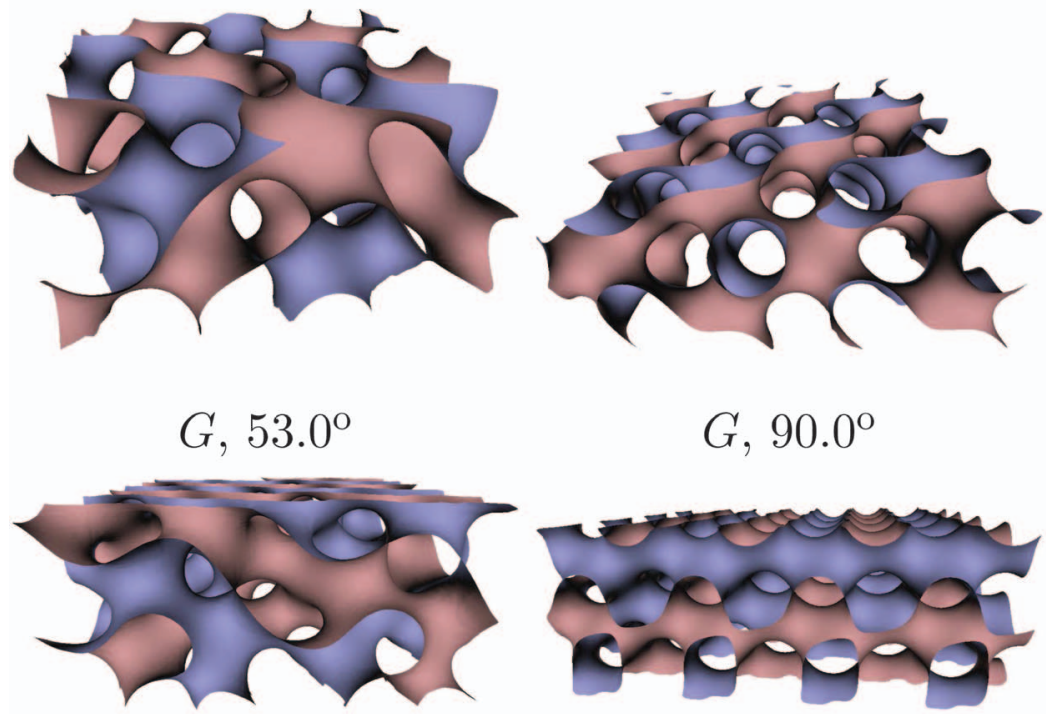

$D, 53.0^{\circ}$

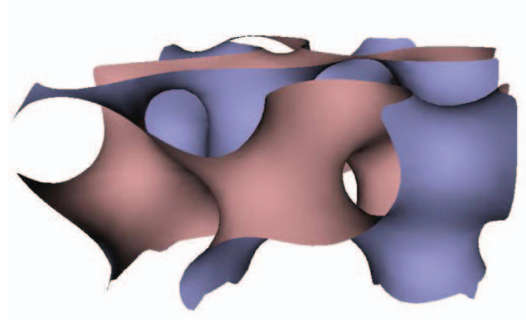

$P, 53.0^{\circ}$

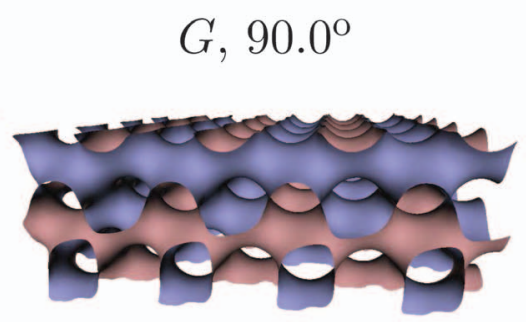

$D, 90.0^{\circ}$

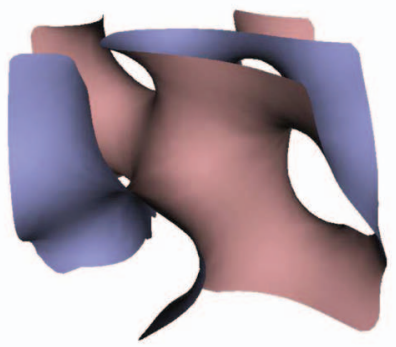

$P, 90.0^{\circ}$
FIG. 7. (Color) Twist grain boundary geometries for the lamellar, gyroid, diamond, and Schwarz $P$ phases for twist angles of $53^{\circ}$ (left) and $90^{\circ}$ (right). See Ref. 43 for movies showing the structure of the monolayer in the grain boundaries in the gyroid phase from different angles. distributions in the bulk are not present in the grain boundaries. The distributions in the grain-boundary regions show an enhancement at low $|K|$ values in comparison to the corresponding distributions in the bulk, signaling the presence of regions more planar than in the bulk. Finally, the Gaussian-curvature distributions in the grain boundaries extend to larger $|K|$ values than the corresponding distributions in the bulk. This can be attributed to the more complex geometry of the surfactant monolayer in the grain boundaries, as in the case of the squared-mean-curvature distributions, but may also signal the appearance of a number of necks, which are slightly narrower than in the bulk.

In the $P$ phase, the behavior is significantly different. The Gaussian-curvature distributions in the original bulk, the final bulk region, and the grain-boundary region for a twist angle of $53^{\circ}$ are shown in Fig. 11. The smaller resolution per unit cell leads to a larger deviation of the distribution in the original bulk from the result obtained from the Weierstrass representation. The distributions in the final bulk and the grain-boundary regions are strikingly similar. Although the peak in the $K$ distribution is not very well resolved in the original bulk, it completely disappears in the final bulk region. The whole distribution shifts to lower $|K|$ values, and in both the final bulk and the grain boundary exhibits a broader tail at higher $|K|$ values. Thus, the geometries of the monolayer in the final bulk and the grain-boundary regions are very similar. The grain boundary grows all the way into the original bulk, and the Schwarz $P$ phase is destroyed.

In the Ginzburg-Landau theory, there are curvature contributions as well as direct interactions between the mono- 


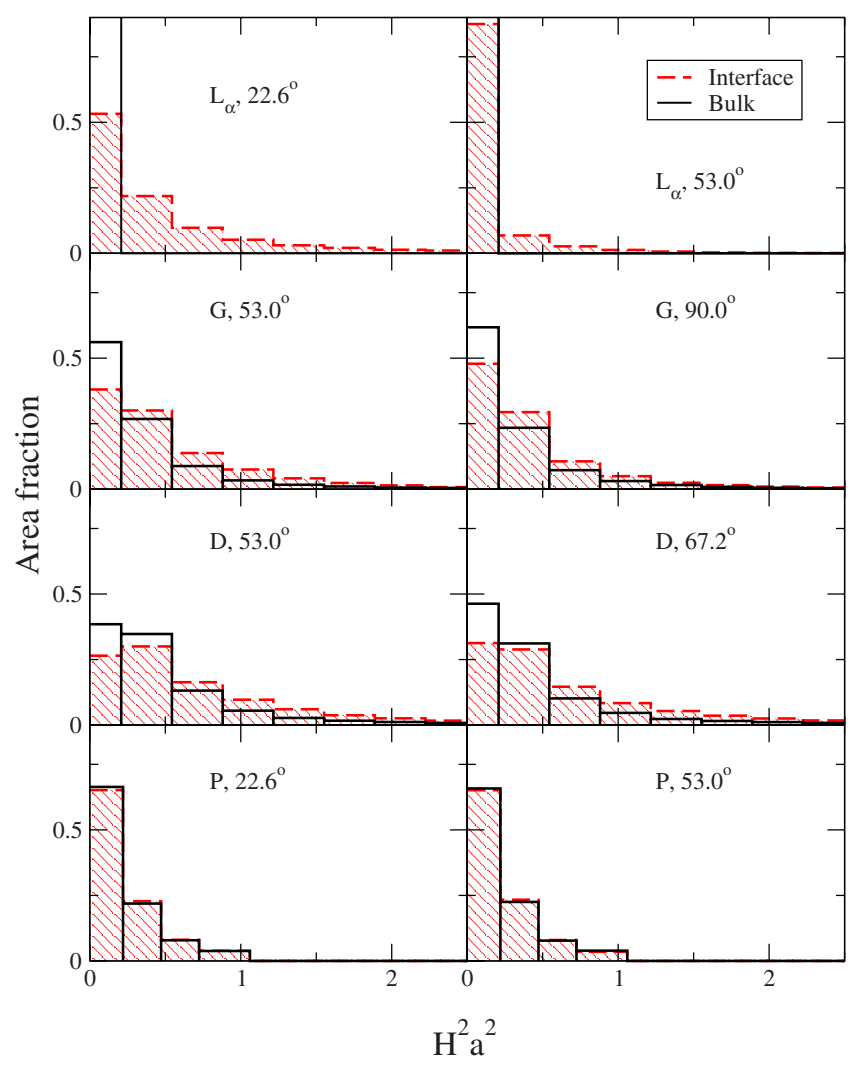

FIG. 8. (Color online) Squared-mean-curvature distributions for the $L_{\alpha}, G$, $D$, and $P$ phases for different twist angles $\alpha$. The histograms are normalized such that the sum of all histogram bins is equal to 1 .

layers. When the curvature energy dominates, $\phi=0$ level surfaces are very close but not identical to minimal surfaces, see Ref. 13. From the squared-mean-curvature distributions we conclude that within the discretization errors of the realspace representation the surfactant monolayer in the grain boundaries is a good approximation of a minimal surface. In the lamellar phase, the twist grain boundaries are well described by the Scherk's minimal surface. The Gaussiancurvature distributions show that in the $G$ and $D$ phases the configuration of the monolayer in the grain boundaries has a significantly different geometry than in the bulk regions. The Schwarz $P$ phase is unstable with the grain boundaries extending all the way into the original bulk regions.

\section{B. Interfacial tension}

\section{Interfacial tension from Ginzburg-Landau theory}

Equation (6) provides a direct method for the determination of interfacial tension from the Ginzburg-Landau theory. In this case, knowledge about the locations of the grain boundaries is not required, since Eq. (6) involves only the free energy of the full system and the free energy of the bulk phase, which fills the same volume.

The interfacial tension as a function of the twist angle determined within the Ginzburg-Landau theory for the lamellar, gyroid, diamond, and Schwarz $P$ phases is shown in Fig. 12.

For all phases the interfacial tension has the same order of magnitude and exhibits a nonmonotonic dependence on the twist angle $\alpha$. In the lamellar phase the interfacial tension

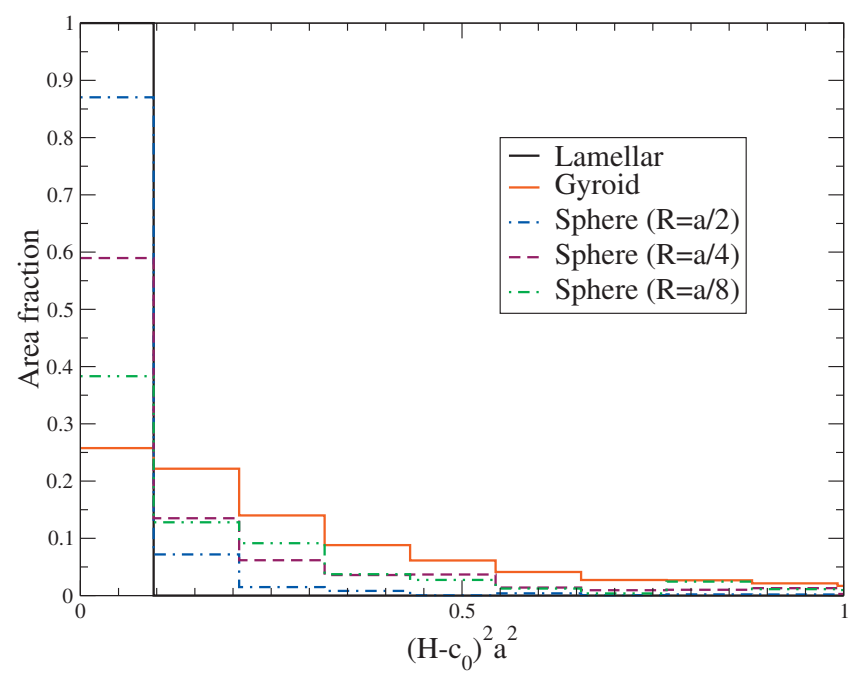

FIG. 9. (Color) Comparison of squared-mean-curvature distributions in the gyroid unit cell (Fig. 1) and spherical caps of radii 1/2,1/4, and 1/8 in units of the gyroid unit cell. More complex geometries lead to broader curvature distributions.

reaches a maximal value at $\alpha \approx 20^{\circ}$ and remains essentially constant above $\alpha \approx 40^{\circ}$. This result is in qualitative agreement with the calculation of Ref. 28 based on the explicit expressions for Scherk's minimal surfaces in combination with bending and compression energies, ${ }^{44}$ where some parameters such as the compression modulus are determined from self-consistent field theory for a block copolymer system. ${ }^{28}$

Interfacial tensions in the $G$ and $D$ phases have similar behavior. There are two maxima at twist angles of about $30^{\circ}$ and $70^{\circ}$, where the interfacial tension is comparable in magnitude to the interfacial tension in the lamellar phase. In the local minima at twist angles of about $50^{\circ}$ the interfacial tension essentially vanishes.

Negative interfacial tension in the Schwarz $P$ phase confirms that in the chosen point of the phase diagram the $P$ phase is not stable with respect to the nucleation of grain boundaries.

The interfacial tension in all cases is very small. The difference in the free energy of the grain boundaries, the thickness of which is determined by the similarity integral (5), and bulk-phase regions of the same volume is of the order of just a few percent at angles corresponding to the maxima of the interfacial tension.

\section{Interfacial tension from geometrical considerations}

The model membrane Hamiltonians introduced in Sec. IV B 2 can be applied for the $G$ and $D$ phases where the locations of the grain boundaries are well defined and the elastic moduli can be calculated such that the bulk structure is a local minimum of the Hamiltonian due to nonvanishing values of the Gaussian curvature. The isosurface $\phi \equiv 0$ determined from the Ginzburg-Landau theory is taken as the geometry of the membrane in these approaches.

We first consider the Hamiltonian (8). The value of $\bar{\kappa}$ sets the normalization. Without loss of generality, we set $\bar{\kappa}=1$ and minimize the Hamiltonian (8) with respect to the 

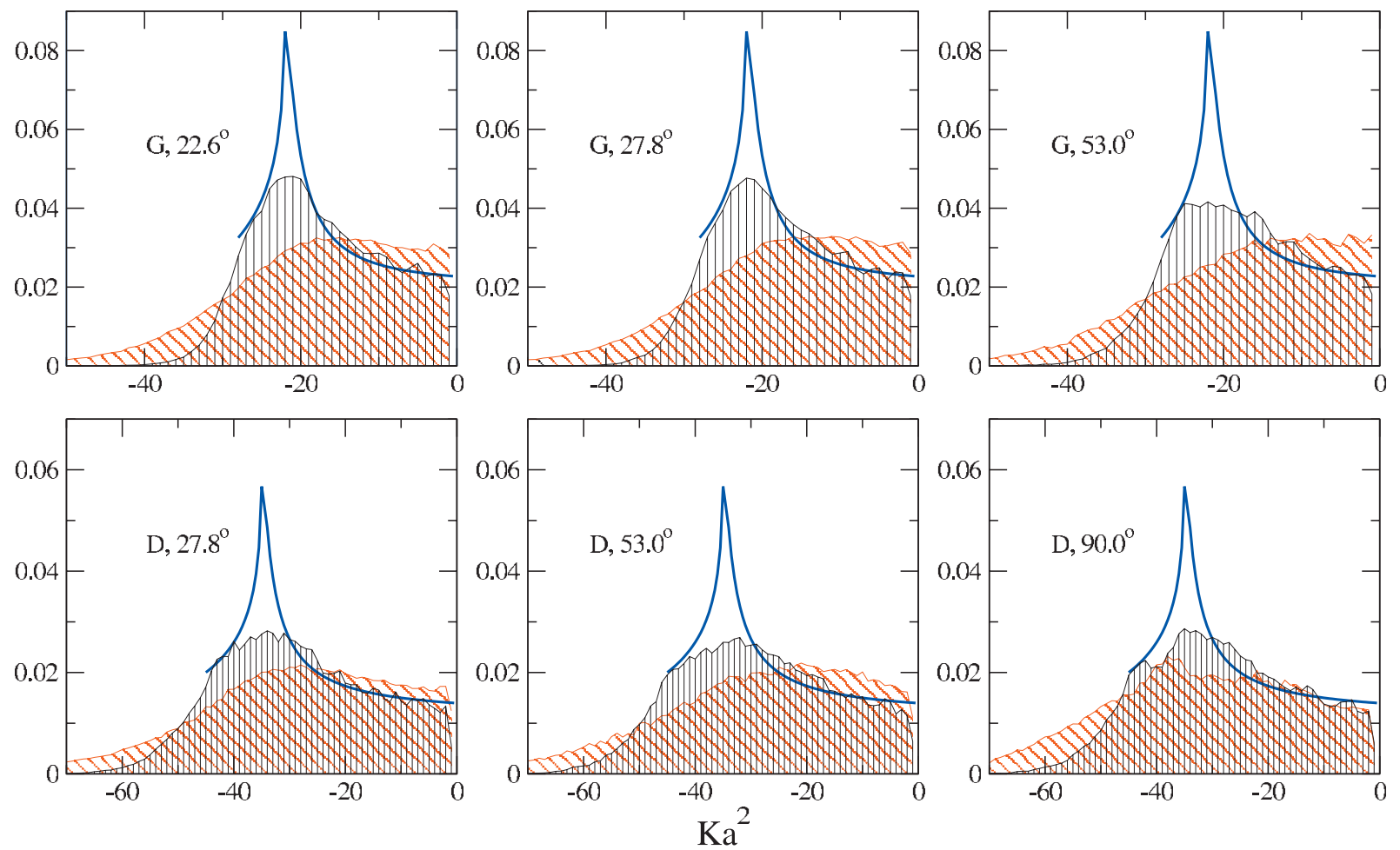

FIG. 10. (Color) Gaussian-curvature distributions for the $G$ and $D$ phases for several twist angles $\alpha$. The histograms are normalized such that the sum of all histogram bins is equal to 1 . The blue solid lines correspond to the exact results obtained from the Weierstrass representation (Ref. 13). The black vertically shaded and red slant shaded histograms correspond to the curvature distributions in the bulk and grain-boundary regions, respectively.

length scales of the $G$ and $D$ unit cells taken from GinzburgLandau theory. This gives $\overline{\bar{\kappa}}=3.113$ for the gyroid and $\overline{\bar{\kappa}}=2.86998$ for the diamond phases, respectively. While these two values of $\overline{\bar{\kappa}}$ are not identical, they are very close. The results for the interfacial tension as a function of the twist angle shown in Fig. 13 are in good qualitative agreement with the results of the Ginzburg-Landau theory shown in Fig. 12. Interfacial tension is of the same order of magnitude for both phases. The two local maxima at twist angles of about $30^{\circ}$ and $70^{\circ}$ are separated by a minimum at about $50^{\circ}$, although the minimum is less pronounced than in the results obtained from the Ginzburg-Landau theory.

Finally, the Hamiltonian (9) is used to determine the interfacial tension in the $G$ and $D$ phases. We first minimize the Hamiltonian for the $G$ and $D$ unit cells for $\overline{\bar{\kappa}}=0$. This gives $\bar{\kappa}=-2.413$ for the $G$ and $\bar{\kappa}=-1.952$ for the $D$ phases, respectively. These values are again not identical but are close. Using the approach described in Sec. IV B 2, we obtain the final set of parameters: $\bar{\kappa}=-1.689, \overline{\bar{\kappa}}=3.756$ for the $G$ phase and $\bar{\kappa}=-1.366, \overline{\bar{\kappa}}=2.801$ for the $D$ phase. Results for the interfacial tension shown in Fig. 14 are in good qualitative agreement with the results obtained from the GinzburgLandau theory and the geometrical approach based on the model Hamiltonian (8). In this case, the minimum of the interfacial tension at twist angles $\alpha \approx 50^{\circ}$ is well represented. Very small negative values of the interfacial tension in $G$ and $D$ phases (Fig. 12) and in the $D$ phase (Fig. 14) at angles around $50^{\circ}$ do not allow to draw a conclusion about the stability of these phases within our numerical accuracy.

\section{SUMMARY AND CONCLUSIONS}

Twist grain boundaries in the lamellar $L_{\alpha}$, gyroid $G$, diamond $D$, and Schwarz $P$ phases have been studied using a Ginzburg-Landau theory of ternary amphiphilic systems. Calculations have been performed for several twist angles for each phase. The surfactant monolayer corresponding to the isosurface $\phi \equiv 0$ has been studied.

In all cases, the squared-mean-curvature and Gaussiancurvature distributions reveal the structure of the surfactant monolayer in the twist grain boundaries to be a good discrete representation of a minimal surface. In the lamellar phase, the monolayer in twist grain boundaries has a geometry very

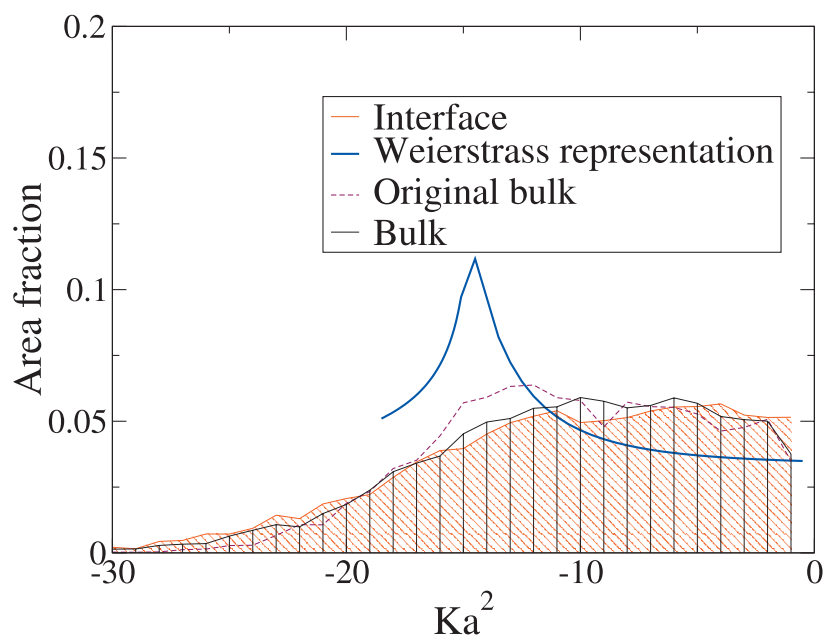

FIG. 11. (Color) Gaussian-curvature distributions for the Schwarz $P$ phase at a twist angle of $53^{\circ}$. 


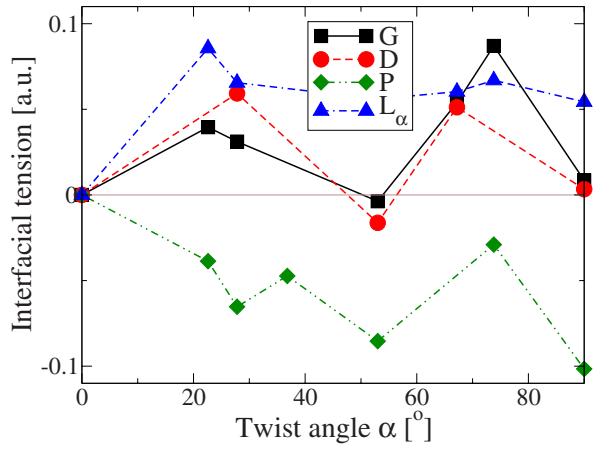

FIG. 12. (Color online) Interfacial tension as a function of the twist angle $\alpha$ for the lamellar, gyroid $G$, diamond $D$, and Schwarz $P$ surfactant phases as extracted from Ginzburg-Landau theory.

similar to the Scherk's minimal surface. The geometry of the monolayer in grain boundaries in $G$ and $D$ phases is more complex. The Gaussian-curvature distributions show significant differences between grain-boundary and bulk regions. The surfactant monolayer in grain boundaries is on average more planar.

The Schwarz $P$ phase is found to be unstable with respect to the nucleation of grain boundaries at the investigated point in the Ginzburg-Landau phase diagram. The similarity integral shows that the grain boundaries grow all the way into the bulk regions. Gaussian-curvature distributions indicate that the surfactant monolayer in the final bulk regions has a geometry very similar to the geometry in the grainboundary regions. This result is not surprising since the Schwarz $P$ phase has a significantly higher free-energy density than the gyroid and diamond phases at the investigated point in the Ginzburg-Landau phase diagram. ${ }^{13}$

Interfacial tension has been determined using two complementary approaches. The first approach is a direct calculation based on the Ginzburg-Landau free-energy functional, where the interfacial tension arises from direct monolayer-monolayer interactions, which set the preferred lamellar spacing, and curvature contributions. The second approach determines interfacial tension from geometrical considerations based on the Canham-Helfrich Hamiltonian for the isosurface $\phi \equiv 0$ determined from the GinzburgLandau theory. The results obtained through both methods are in good qualitative agreement.

Interfacial tension is found to have the same order of

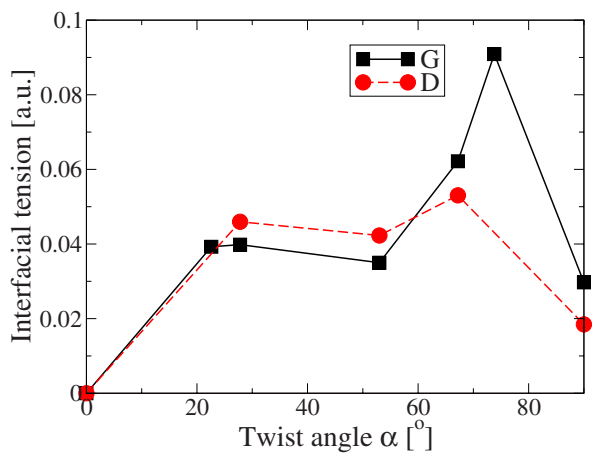

FIG. 13. (Color online) Interfacial tension as a function of the twist angle $\alpha$ for the gyroid $G$ and diamond $D$ surfactant phases as determined using the model membrane Hamiltonian (8).

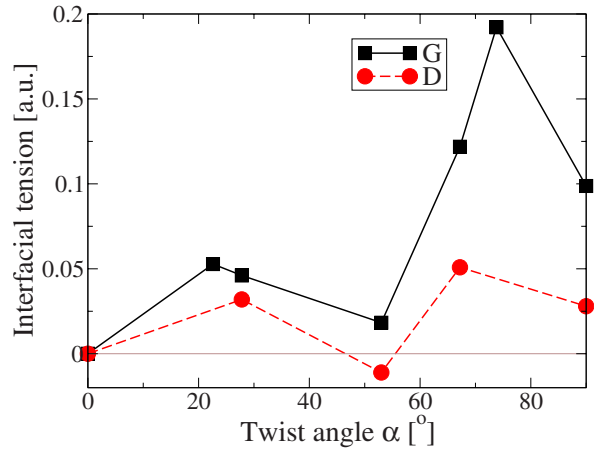

FIG. 14. (Color online) Interfacial tension as a function of the twist angle $\alpha$ for the gyroid $G$ and diamond $D$ surfactant phases as determined using the model membrane Hamiltonian (9).

magnitude and a nonmonotonic dependence on the twist angle for all phases. Interfacial tension in the lamellar phase has a maximum at twist angles of about $20^{\circ}$ and is essentially constant at twist angles greater than $40^{\circ}$. Interfacial tension in the gyroid and diamond phases has two maxima at twist angles of about $30^{\circ}$ and $70^{\circ}$ and a local minimum at twist angles of about $50^{\circ}$ where the interfacial tension essentially vanishes. Because the interfacial tension is very small, the density of grain boundaries should be high in these surfactant phases.

${ }^{1}$ J. Tabony, Nature (London) 319, 400 (1986).

${ }^{2}$ R. Strey, Colloid Polym. Sci. 272, 1005 (1994).

${ }^{3}$ W. M. Gelbart, A. Ben-Shaul, and D. Roux, Micelles, Membranes, Microemulsions, and Monolayers (Springer, New York, 1994).

${ }^{4}$ K. Fontell, Colloid Polym. Sci. 268, 264 (1990).

${ }^{5}$ J. M. Seddon, Biochim. Biophys. Acta 1031, 1 (1990).

${ }^{6}$ V. Luzzati, R. Vargas, P. Mariani, A. Gulik, and H. Delacroix, J. Mol. Biol. 229, 540 (1993).

${ }^{7}$ G. Gompper and M. Schick, in Self-assembling Amphiphilic Systems of Phase Transitions and Critical Phenomena, edited by C. Domb and J. L. Lebowitz (Academic, New York, 1994), Vol. 16.

${ }^{8}$ U. S. Schwarz and G. Gompper, in Morphology of Condensed Matter: Physics and Geometry of Spatially Complex Systems, edited by K. R. Mecke and D. Stoyan (Springer, New York, 2002), Vol. 600, pp. 107-151.

${ }^{9}$ S. Komura, J. Phys.: Condens. Matter 19, 463101 (2007).

${ }^{10}$ S. T. Hyde, S. Andersson, K. Larsson, S. Lidin, T. Landh, Z. Blum, and B. W. Ninham, The Language of Shape (Elsevier, New York, 1997).

${ }^{11}$ D. M. Anderson, S. M. Gruner, and S. Leibler, Proc. Natl. Acad. Sci. U.S.A. 85, 5364 (1988).

${ }^{12}$ Z.-G. Wang and S. A. Safran, Europhys. Lett. 11, 425 (1990).

${ }^{13}$ U. S. Schwarz and G. Gompper, Phys. Rev. E 59, 5528 (1999).

${ }^{14}$ U. S. Schwarz and G. Gompper, J. Chem. Phys. 112, 3792 (2000).

${ }^{15}$ K. Grosse-Brauckmann, J. Colloid Interface Sci. 187, 418 (1997).

${ }^{16}$ G. E. Schröder-Turk, A. Fogden, and S. T. Hyde, Eur. Phys. J. B 59, 115 (2007).

${ }^{17}$ T. Landh, FEBS Lett. 369, 13 (1995).

${ }^{18}$ S. G. Attard, J. C. Glyde, and C. G. Göltner, Nature (London) 378, 366 (1995).

${ }^{19}$ E. Pebay-Peyroula, G. Rummel, J. P. Rosenbusch, and E. M. Landau, Science 277, 1676 (1997).

${ }^{20}$ R. Bruinsma, J. Phys. II 2, 425 (1992).

${ }^{21}$ R. R. Netz, D. Andelman, and M. Schick, Phys. Rev. Lett. 79, 1058 (1997).

${ }^{22}$ S. Villain-Guillot, R. R. Netz, D. Andelman, and M. Schick, Physica A 249, 285 (1998).

${ }^{23}$ K. Yamada and S. Komura, J. Phys.: Condens. Matter 20, 155107 (2008).

${ }^{24}$ K. Yamada and T. Ohta, J. Phys. Soc. Jpn. 76, 084801 (2007).

${ }^{25}$ H. Dohi, H. Kimura, M. Kotani, T. Kaneko, T. Kitaoka, T. Nishi, and H. Jinnai, Polym. J. (Tokyo, Jpn.) 39, 749 (2007). 
${ }^{26}$ P. Pieranski, EPL 81, 66001 (2008).

${ }^{27}$ Y. Tsori, D. Andelman, and M. Schick, Phys. Rev. E 61, 2848 (2000).

${ }^{28}$ D. Duque and M. Schick, J. Chem. Phys. 113, 5525 (2000).

${ }^{29}$ H. Jinnai, K. Sawa, and T. Nishi, Macromolecules 39, 5815 (2006).

${ }^{30}$ Y. Cohen, R. J. Albalak, B. J. Dair, M. S. Capel, and E. L. Thomas, Macromolecules 33, 6502 (2000).

${ }^{31}$ N. González-Segredo and P. V. Coveney, Europhys. Lett. 65, 795 (2004).

${ }^{32}$ G. Gompper and M. Schick, Phys. Rev. Lett. 65, 1116 (1990).

${ }^{33}$ K. Chen, C. Jayaprakash, R. Pandit, and W. Wenzel, Phys. Rev. Lett. 65, 2736 (1990).

${ }^{34}$ G. Gompper and M. Kraus, Phys. Rev. E 47, 4289 (1993).

${ }^{35}$ P. B. Canham, J. Theor. Biol. 26, 61 (1970).

${ }^{36}$ W. Helfrich, Z. Naturforsch [C] 28, 693 (1973).
${ }^{37}$ G. Gompper and S. Zschocke, Europhys. Lett. 16, 731 (1991).

${ }^{38}$ G. Gompper and S. Zschocke, Phys. Rev. A 46, 4836 (1992).

${ }^{39}$ W. Góźdź and R. Hołyst, Phys. Rev. E 54, 5012 (1996).

${ }^{40}$ G. Gompper and M. Kraus, Phys. Rev. E 47, 4301 (1993).

${ }^{41}$ H. G. Schnering and R. Nesper, Z. Phys. B 83, 407 (1991).

${ }^{42}$ S. Popinet, The GNU Triangulated Surface Library. Available from http:// gts.sourceforge.net.

${ }^{43}$ See EPAPS Document No. E-JCPSA6-130-022912 for movies showing the structure of the monolayer in twist grain boundaries in the gyroid phase from different angles. For more information on EPAPS, see http:// www.aip.org/pubservs/epaps.html.

${ }^{44}$ R. D. Kamien and T. C. Lubensky, Phys. Rev. Lett. 82, 2892 (1999). 